\title{
A Multi-objective Mathematical Model Considering Economic and Social Criteria in Dynamic Cell Formation
}

\author{
Farzad Niakan, Armand Baboli, Thierry Moyaux, and Valerie Botta-Genoulaz \\ Université de Lyon, INSA-Lyon, DISP EA4570, 69621 VILLEURBANNE, France \\ \{Farzad.Niakan, Armand.Baboli, Thierry.Moyaux, \\ Valerie.Botta\}@insa-lyon.fr
}

\begin{abstract}
This paper addresses a Dynamic Cellular Manufacturing Systems (DCMS) problem considering both economic and social criteria, that is, the problem deals with the minimization of the total costs and maximization of social issues. We develop a bi-objective mathematical model of this problem in order to capture the trade-off between these two objectives. The strategic decisions considered in the model define the configuration of cells and part-families in each period. In order to solve our model, we design a new Non-dominated Sorting Genetic Algorithm (NSGA-II) as a meta-heuristic method. Our approach is illustrated on two samples of problems randomly generated.
\end{abstract}

Keywords: Dynamic cellular manufacturing system, Social responsibility, Multi-objective optimization model.

\section{Introduction}

Now a day most industries face with dynamic production because of the rapid product changes and reducing the life cycle of products. This observation leads to reorganize the manufacturing cells, from one period to each other, during planning horizons (several periods). On the other hand, in most of cell formation methods, the decision affect the reconfiguration of cells and definition of the part families just for a singleperiod which might not be optimal in another period. Therefore, this reason motivates researchers and manufacturers to study cellular manufacturing system (CMS) under dynamic condition that is named as Dynamic Cellular Manufacturing System (DCMS). Moreover, pressures from NGOs, social communities and media to respect social issues caused a lot of damages to some of well-known corporations such as McDonalds, Mitsubishi, Monsanto, Nestlé, Nike, Shell and Texaco. Corporate Social Responsibility (CSR) deals with effect of corporate activities on different social entities such as environment preservation, human rights, labor safety, etc. [1]. As the CSR aims to make trade-off between economical aspect and social issues to provide more welfare for the whole society. Despite the importance of CSR, social issues is one of the neglected affaires in the previous study involved in DCMS. This paper presents a new optimization model for DCMS, in which a trade-off between economic and social objectives is made. 


\section{Literature Review}

Many researches are related to DCMS, hence, we describe more recent studies here. Rheault et al. [2] presented the concept of dynamic environment in cell formation problem for the first time. Schaller et al. [3] developed a new model in DCMS to minimize Configuration and inventory cost of the system. They also proposed a Lagrangian relaxation to obtain the lower bound for their problem. Chen and Cao [4] proposed a new mathematical model in DCMS by considering inventory level. They solve their presented model by Tabu Search (TS) as a well-known algorithm. Tavakkoli-Moghaddam et al. [5] formulated a new nonlinear integer mathematical model in DCMS. The authors minimized constant and variable costs of machine and inter-cell movement costs as objective function. They solved their models by several meta-heuristic methods such as Genetic Algorithm (GA), Tabu Search (TS) and Simulated Annealing (SA) and compared obtained solutions to determine best method. Defersha and Chen [6] proposed a new comprehensive model in DCMS. They considered alternative routings, lot splitting and sequence of operations as well as workload balancing in their model. Safaei et al. [7] presented a fuzzy programming approach in CMS with dynamic and uncertain condition. The authors in this study assumed that demand of parts and availability of each machine have uncertain and fuzzy condition. Safaei and Tavakkoli-Moghaddam [8] formulated a new mathematical model in DCMS by focusing on the operational aspect of the cell formation problem. They made trade-off between production and outsourcing costs on the re-configuration of the system. Javadian et al. [9] proposed a multi-objective mathematical model in order to minimize the variation of cell load and various costs simultaneously. In order to solve model and obtain optimal Pareto frontier, they designed a non-dominated sorting genetic algorithm (NSGA-II). Saxena and Jain [10] proposed a new mixed-integer nonlinear mathematical model in DCMS to integrate important attributes of manufacturing such as the effects of machine breakdowns, production time loss cost effect, inventory holding, lot splitting; alternative process plan and parts sequence of operation. Kia et al. [11] developed a novel mixed-integer non-linear programming model in design of CMS with dynamic condition. The objective function of their model minimize total cost of each period such as costs of intra/inter-cell movement, machine relocation, machine procurement, machine overhead and machine processing. Majazi Dalfard [12] described a new nonlinear integer programming model for DCMS. The contribution of this paper is mentioned in two ways, the first one is related to apply implementation of the idea of more material flow in shorter distance in configuration of the cells and the second one is the proposition of new simulated annealing embedded in branch and cut as solving method. This investigation of literature shows that social issues is a neglected issue in DCMS while only economical aspect have been considered as objective of researches most of the time. Thus, in order to eliminate this deficiency, we develop a new multi-objective mathematical model for DCMS configuration by also considering social aspects. 


\section{Problem Description}

In this section, we present a new bi-objective mathematical model in order to make a trade-off between two important objectives in DCMS. The first objective function is the minimization of relevant costs such as machine fixed and variable costs, inter-cell movement costs, intra-cell movement costs, machine procurement costs, machine relocation costs, salary of labors, etc. The second objective function is the maximization of the social criteria by increasing of job opportunities and decreasing of potential machines hazards for labor during the planning horizon.

Generally, the complex nature and interdependencies of social criteria make it difficult to measure them. In this study, an attempt has been made to select and extract social issues from ISO 26000 and GRI 2011 (credible sustainability reporting framework) [13],[14] that are closely related to DCMS problem and can be simply calculated by the mathematical model. As a result, (i) the number of job opportunities created and (ii) the number of potential hazards of machines were selected as social issues of our model. The first, social issue represents the variation of job opportunities, created due to the hiring and firing of labor and resulting in buying, selling and determining of time capacity of machines. The next one measures the average fraction of potential hazards of each machine, which is how many injuries, illnesses and damages each machine causes for labors according to ergonomic criteria. This value is a continuous number between 0 (the safest) and 1 (most dangerous) and can be defined by the Decision Makers (DM). For this objective, they can use a safety checklist, talking to workers, reviewing the manufacturer's information, and checking the injury and incident reports of each machine. Due to differences in the measuring units of the considered social criteria, the weighting method (normalized weighted) was applied in order to aggregate them as one objective. The main aim of this research work is to study the effects of social criteria on DCMS configuration. Therefore, other social issues may be replaced or involved in the model, based on a normalized weighted method.

\subsection{Notations}

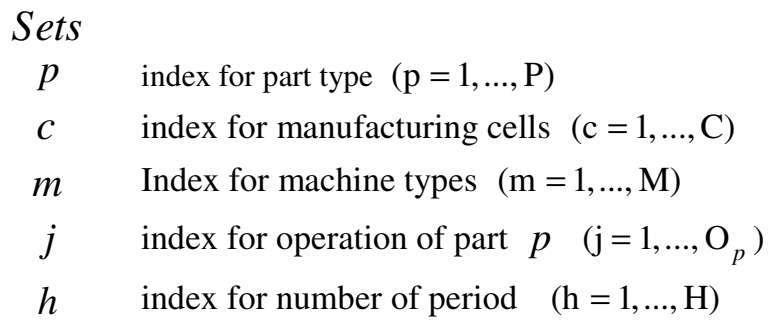

\section{Parameters}

$D_{p h} \quad$ demand for part $p$ in period $h$

$B_{p}^{\text {inter }} \quad$ batch size for inter-cell movement of part $p$

$B_{p}^{\text {int } r a} \quad$ batch size for intra-cell movement of part $p$ 
$\gamma_{p}^{\text {inter }} \quad$ inter-cell movement cost per batch

$\gamma_{p}^{\text {int } r a}$ intra-cell movement cost per batch. It is assumed that $\forall p\left(\gamma_{p}^{\text {int } r a} / B_{p}^{\text {int } r a}\right) \prec\left(\gamma_{p}^{\text {inter }} / B_{p}^{\text {inter }}\right)$

$\alpha_{m h} \quad$ fixed cost of machine type $m$ in period $h$

$\beta_{m h} \quad$ variable cost of machine type $m$ in period $h$ per unit of time

$\delta_{m h} \quad$ relocation cost of machine type $m$ in period $h$

$T_{m h} \quad$ time-capacity of machine type $m$ in period $h$

$U B \quad$ maximum number of cells

$L B \quad$ minimum number of cells

$L_{m} \quad$ amount of labor that is required for machine type $m$

$\varphi_{m h} \quad$ purchase cost of machine type $m$ in period $h$

$\omega_{m h} \quad$ marginal revenue from selling machine type $m$ in period $h$

$S_{m} \quad$ average fraction of potentially hazardous of machine type $m$

$\theta_{h d}$ normalized weighting factor of the total number of potentially hazardous of machine type $m$

$\theta_{j o} \quad$ normalized weighting factor of the total number of job opportunities created

$\lambda_{h} \quad$ maximum percentage of variation labor level in period $h$

$s a_{m h}^{L} \quad$ salary of labor on machine type $m$ in period $h$

$t_{j p m} \quad$ time required to process operation $j$ of part $p$ on machine type $m$

$a_{j p m} \quad 1$, if operation $j$ of part $p$ can be done on machine type $m ; 0$ otherwise

\section{Variables} $N_{m c h} \quad h \quad$ number of machine type $m$ assigned to cell $c$ at the beginning of period

$K_{m c h}^{+} \quad$ number of machine type $m$ added to cell $c$ at the beginning of period $h$

$K_{m c h}^{-} \quad$ number of machine type $m$ removed to cell $c$ at the beginning of period $h$

$I_{m h}^{+} \quad$ number of machine type $m$ purchased at the beginning of period $h$

$I_{m h}^{-} \quad$ number of machine type $m$ sold at the beginning of period $h$

$h i_{h} \quad$ number of labor hired in period $h$

$f i_{h} \quad$ number of labor fired in period $h$

$x_{\text {jpmch }} 1$, if operation $j$ of part $p$ is performed in cell $c$ in period $h$ by machine type $m$; 0 otherwise 


\subsection{Mathematical Formulation}

$$
\begin{aligned}
& \text { Min } Z_{1}=\sum_{h=1}^{H} \sum_{m=1}^{M} \sum_{c=1}^{C} \alpha_{m h} N_{m c h}+\sum_{h=1}^{H} \sum_{m=1}^{M} \sum_{c=1}^{C} \sum_{p=1}^{P} \sum_{j=1}^{O_{p}} \beta_{m h} D_{p h} t_{j p m} x_{j p m c h} \\
& +\frac{1}{2} \sum_{h=1}^{H} \sum_{p=1}^{P} \gamma_{p}^{\text {inter }}\left[\frac{D_{p h}}{B_{p}^{\text {inter }}}\right] \times \sum_{j=1}^{o_{p}-1} \sum_{c=1}^{C}\left|\sum_{m=1}^{M} x_{(j+1) p m c h}-\sum_{m=1}^{M} x_{j p m c h}\right| \\
& +\frac{1}{2} \sum_{h=1}^{H} \sum_{p=1}^{P} \gamma_{p}^{\text {int } r a}\left[\frac{D_{p h}}{B_{p}^{\text {int } r a}}\right] \times \sum_{j=1}^{o_{p}-1} \sum_{c=1}^{c}\left(\sum_{m=1}^{M}\left|x_{(j+1) p m c h}-x_{j p m c h}\right|-\left|\sum_{m=1}^{M} x_{(j+1) p m c h}-\sum_{m=1}^{M} x_{j p m c h}\right|\right) \\
& +\sum_{h=1}^{H} \sum_{m=1}^{M} \sum_{c=1}^{C} N_{m c h} L_{m} S a_{m h}^{L}+\frac{1}{2} \sum_{h=1}^{H} \sum_{m=1}^{M} \sum_{c=1}^{C} \delta_{m h}\left(K_{m c h}^{+}+K_{m c h}^{-}\right) \\
& +\sum_{h=1}^{H} \sum_{m=1}^{M} I_{m h}^{+} \varphi_{m h}-\sum_{h=1}^{H} \sum_{m=1}^{M} I_{m h}^{-} \omega_{m h} \\
& \operatorname{Max} Z_{2}=\theta_{j o}\left(\sum_{h=1}^{H} h i_{h}-\sum_{h=1}^{H} f i_{h}\right)-\theta_{h d}\left(\sum_{h=1}^{H} \sum_{m=1}^{M} \sum_{c=1}^{C} N_{m c h} S_{m}\right) \\
& S . t .
\end{aligned}
$$

S.t.

$$
\sum_{c=1}^{C} \sum_{m=1}^{H} a_{j p m} x_{j p m c h}=1
$$

$x_{j p m c h} \leq a_{j p m}$

$\forall j, p, c, m, h$

$\sum_{p=1}^{P} \sum_{j=1}^{O_{p}} D_{p h} t_{j p m} x_{j p m c h} \leq T_{m h} N_{m c h}$

$\forall m, c, h$

$N_{m c(h-1)}+K_{m c h}^{+}-K_{m c h}^{-}=N_{m c h}$

$\forall m, c, h$

$\sum_{c=1}^{C} N_{m c(h-1)}+I_{m h}^{+}-I_{m h}^{-}=\sum_{c=1}^{C} N_{m c h}$

$\forall m, h$

$\sum_{m=1}^{M} N_{m c h} \leq U B$

$\forall c, h$

$\sum_{m=1}^{M} N_{m c h} \geq L B$

$\forall c, h$

$\sum_{m=1}^{M} I_{m h}^{+} L_{m} \leq h i_{h}$

$\forall h$

$\sum_{m=1}^{M} I_{m h}^{-} L_{m} \leq f i_{h}$

$\forall h$ 


$$
\begin{aligned}
& \left|\sum_{m=1}^{M}\left[\left(I_{m h}^{+}-I_{m h}^{-}\right) L_{m}\right]\right| \leq\left(\sum_{m=1}^{M}\left(\sum_{c=1}^{C} N_{m c(h-1)}\right) L_{m}\right) \lambda_{h} \quad \forall h \\
& x_{j p m c h} \in\{0,1\}, N_{m c h}, K_{m c h}^{+}, K_{m c h}^{-} \geq 0 \text { and int eger } \\
& I_{m h}^{+}, I_{m h}^{-}, h i_{h}, f i_{h} \geq 0 \text { and int eger }
\end{aligned}
$$

Objective function (1) represents the minimization of total costs and includes eight terms: (i) machine fixed costs, (ii) variable machine cost, (iii) costs of the movements of parts between cells (inter-cell), (iv) costs of the movements of parts inside of cells (intra-cell), (v) wages or salaries, (vi) machine relocation cost, (vii) machine procurement cost and (viii) revenue from sales of the machines. Objective function (2) maximizes the social issues of DCMS during the planning horizon, and includes the maximization of job opportunities and minimization of the potentially hazardous of machines. Constraint (3) ensures that each operation of a part is assigned to just one machine and one cell. Constraint (4) guarantees that the process of each part assigned to the machines that can be done it. Constraint (5) ensures that the time capacity of each machine is not exceeded. Constraints (6) and (7) ensure that in each planning period the number of machines is balanced. Constraints (8) and (9) determine the maximum and minimum cell sizes according to the defined upper and lower bound. Constraints (10) and (11) calculate the number of labors that are respectively hired and fired. Constraint (12) guarantees maximum variation of the labor level in each period. Constraint (13) states that decision variables are non-negative, binary and/or integer.

\section{Resolution Method}

Since such a cell formation problem is defined as an NP-hard optimization problem frequently by researchers. In order to cope with complexity of the proposed model, a Non-Dominated Sorting Genetic Algorithm II (NSGA-II) [15], is designed as a metaheuristic which is belonged to evolutionary algorithms. This algorithm starts with an initial solution (population) that is randomly generated and in each iteration new solutions (children) are produced from existing solutions with crossover and mutation operators. It should be noted that parents are selected by a binary tournament selection process. In each iteration, the objective value of each solution is compared against two strategies, namely, ranking and crowding distance. In order to improve the efficiency of the proposed NSGA-II algorithm and find best results, a Taguchi design is applied as a statistical technique to find the best combination of the parameters of NSGA-II. Hence, Npop (number of members in each population), MaxIt (number of iterations), CrR (crossover rate) and MuR (mutation rate) are tuned as four vital parameters of algorithm. Table 1 shows the results obtained here. 
Table 1. Taguchi result for NSGA-II parameters

\begin{tabular}{|c|c|c|c|c|}
\hline Parameters & Npop & MaxIt & CrR & $M u R$ \\
\hline Tuned value & 40 & 50 & 0.8 & 0.3 \\
\hline
\end{tabular}

\section{Computational Result}

In order to verify the performance and applicability of our proposed mathematical model two sets of different test problem in deferent size are generated:

Table 2. The size of samples

\begin{tabular}{ccc}
\hline & $\mathrm{P} 1$ & $\mathrm{P} 2$ \\
\cline { 2 - 3 } & $5 \times 6 \times 6 \times 4 \times 5$ & $9 \times 8 \times 8 \times 7 \times 7$ \\
\hline
\end{tabular}

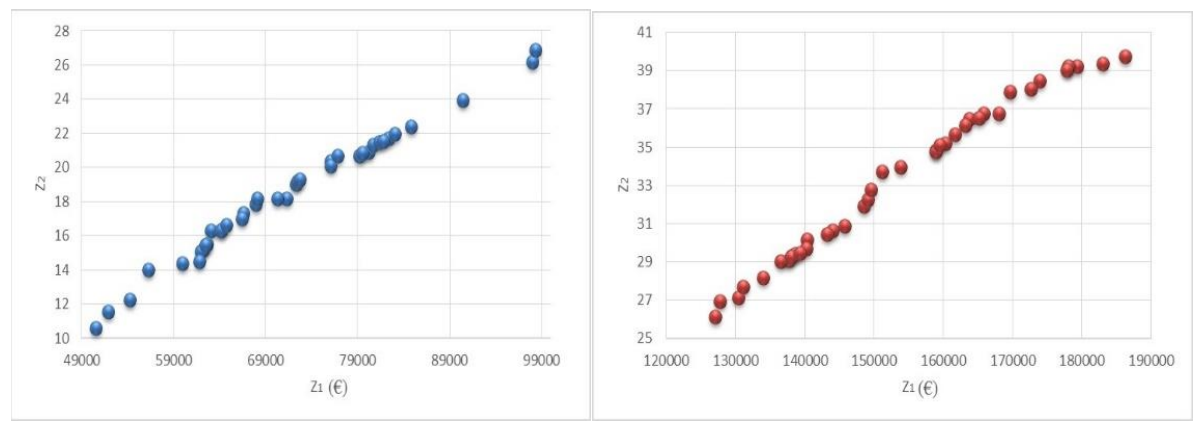

Fig. 1. Optimal Pareto Frontier for P1 (blue) \& P2 (Red)

The generated test problems are solved by proposed NSGA-II algorithm which is developed by using MATLAB 2012a on a computer with Intel Core i5, 2.27 GHz and 4 GB RAM (See Figure 1).

Table 3. Computational Times (Sec)

\begin{tabular}{lcc}
\hline & P1 & P2 \\
\cline { 2 - 3 } Computational Time & 287.275 & 417.829 \\
\hline
\end{tabular}

Figure 1 shows the optimal Pareto frontier and a set of solutions can be obtained from trade-off between minimizing total costs $\left(Z_{1}\right)$ and maximizing of social criteria $\left(\mathrm{Z}_{2}\right)$. According to the priority of each objective the solution can be selected by DM. For each solution, we can have all information about the total cost of system, social issues, configuration and/or modification in each cell, part families, human resources, processing rout, etc. The analysis of optimal Pareto frontiers shows that the maximum of the social criteria $\left(\mathrm{Z}_{2}\right)$ can be obtain by increasing the total cost $\left(\mathrm{Z}_{1}\right)$ by about $90 \%$ in problem $\mathrm{P} 1$ and $60 \%$ in $\mathrm{P} 2$ with regard to the best economical solution. 


\section{Conclusion}

In this paper, we propose a new bi-objective mathematical model in DCMS by considering social and economic criteria. Optimizing both criteria simultaneously requires making a trade-off between them. Due to the complexity of the problem, a NSGA-II algorithm is developed to solve the model and obtain Pareto frontier for the generated test problems. The proposed DCMS configuration model can be applied in batch production industries, such as part suppliers in automotive industry as well as small household electrical products.

Acknowledgment. The authors gratefully acknowledge the scientific and financial supports of Region Rhône-Alpes, France, ARC8 in developing this paper.

\section{References}

1. Carter, C.R., Jennings, M.M.: Social responsibility and supply chain relationships. Transp. Res. Part E Logist. Transp. Rev. 38, 37-52 (2002)

2. Rheault, M., Drolet, J.R., Abdulnour, G.: Physically reconfigurable virtual cells: A dynamic model for a highly dynamic environment. Comput. Ind. Eng. 29, 221-225 (1995)

3. Schaller, J., Selçuk Erengüç, S., Vakharia, A.: A methodology for integrating cell formation and production planning in cellular manufacturing. Ann. Oper. Res. 77, 1-21 (1998)

4. Chen, M., Cao, D.: Coordinating production planning in cellular manufacturing environment using Tabu search. Comput. Ind. Eng. 46, 571-588 (2004)

5. Tavakkoli-Moghaddam, R., Aryanezhad, M.B., Safaei, N., Azaron, A.: Solving a dynamic cell formation problem using metaheuristics. Appl. Math Comput. 170, 761-780 (2005)

6. Defersha, F.M., Chen, M.: A comprehensive mathematical model for the design of cellular manufacturing systems. Int. J. Prod. Econ. 103, 767-783 (2006)

7. Safaei, N., Saidi-Mehrabad, M., Babakhani, M.: Designing cellular manufacturing systems under dynamic and uncertain conditions. J. Intell. Manuf. 18, 383-399 (2007)

8. Safaei, N., Tavakkoli-Moghaddam, R.: Integrated multi-period cell formation and subcontracting production planning in dynamic cellular manufacturing systems. Int. J. Prod. Econ. 120, 301-314 (2009)

9. Javadian, N., Aghajani, A., Rezaeian, J., Ghaneian Sebdani, M.: A multi-objective integrated cellular manufacturing systems design with dynamic system reconfiguration. Int. J. Adv. Manuf. Technol. 56, 307 (2011)

10. Saxena, L., Jain, P.: Dynamic cellular manufacturing systems design-a comprehensive model. Int. J. Adv. Manuf. Technol. 53, 11-34 (2011)

11. Kia, R., Baboli, A., Javadian, N., et al.: Solving a group layout design model of a dynamic cellular manufacturing system with alternative process routings, lot splitting and flexible reconfiguration by simulated annealing. Comput. Oper. Res. 39, 2642-2658 (2012)

12. Majazi Dalfard, V.: New mathematical model for problem of dynamic cell formation based on number and average length of intra and intercellular movements. Appl. Math Model 37, 1884-1896 (2013)

13. ISO, Final Draft International standard ISO/FDIS 26000. Guid. Soc. Responsib (2010)

14. GRI, Sustainability Reporting Guidelines. version 3.1 (2011)

15. Deb, K., Pratap, A., Agarwal, S., Meyarivan, T.: A fast and elitist multiobjective genetic algorithm: NSGA-II. Evol. Comput. IEEE Trans. 6, 182-197 (2002) 